\title{
Sur une formule classique
}

\author{
A Shôkichi Iyanaga en toute amitié \\ par André WEIL
}

(Reçu le 7 juin, 1967)

Dans un mémoire célèbre ([2]), Hecke a établi que les séries de Dirichlet satisfaisant à un certain type d'équation fonctionnelle correspondent au moyen de la transformation de Mellin à certains types de formes modulaires; divers travaux, et tout particulièrement ceux de H. Maass, ont considérablement étendu la portée de cette méthode. D’autre part, on sait maintenant (cf. [4]) que, même sans quitter le cadre des formes modulaires usuelles, la méthode de Hecke peut être appliquée à des problèmes plus généraux que ceux qu'il avait envisagés lui-même, et tout indique qu'on peut encore aller beaucoup plus loin dans les voies ainsi tracées.

Du point de vue de Hecke, il s'agissait avant tout de ramener la recherche de séries de Dirichlet satisfaisant à des équations fonctionnelles à celle des formes modulaires correspondantes, considérées comme mieux connues. Mais la théorie a maintenant fait assez de progrès pour qu'on puisse aussi appliquer utilement les mêmes résultats en sens inverse, et mettre au service de la théorie des fonctions automorphes nos connaissances sur les séries de Dirichlet. Mon propos ici est seulement d'illustrer ce principe au moyen d'un exemple particulièrement simple, et que sans doute Hecke a dû connaître, bien que je n'en aie pas rencontré de mention explicite chez lui ni chez ses successeurs.

Considérons les fonctions

$$
\varphi(s)=\zeta(s) \zeta(s+1), \quad \Phi(s)=(2 \pi)^{-s} \Gamma(s) \varphi(s) .
$$

L'équation fonctionnelle de la fonction zêta, jointe aux propriétés classiques de la fonction gamma, donne aussitôt pour $\Phi$ 1'“équation fonctionnelle” :

$$
\Phi(s)=\Phi(-s) \text {. }
$$

Il est immédiat que $\Phi$ a un pôle double en $s=0$, des pôles simples en $s= \pm 1$, est holomorphe partout ailleurs, et est bornée pour $\sigma \leqq \operatorname{Re}(s) \leqq \sigma^{\prime}, \operatorname{Im}(s) \geqq \varepsilon$, quels que soient $\sigma, \sigma^{\prime}$ et $\varepsilon>0$. Il est clair aussi que $\Phi$ a le résidu $\zeta(2) / 2 \pi$ $=\pi / 12$ en $s=1$, le résidu $-\pi / 12$ en $s=-1$, et que $\Phi(s)+1 / 2 s^{2}$ est holomorphe en $s=0$.

La fonction $\varphi$ est évidemment donnée, pour $\operatorname{Re}(s)>1$, par la série de 
Dirichlet

$$
\varphi(s)=\sum_{m, n=1}^{\infty} \frac{m^{-1}}{(m n)^{s}} .
$$

La série de puissances en $q=e^{2 \pi i \tau}$ qui a les mêmes coefficients est donc:

$$
F(\tau)=\sum_{m, n=1}^{\infty} m^{-1} q^{m n}=\sum_{n=1}^{\infty}\left(\sum_{m=1}^{\infty} m^{-1}\left(q^{n}\right)^{m}\right)=-\sum_{n=1}^{\infty} \log \left(1-q^{n}\right) .
$$

On reconnaît là, à un terme près, le logarithme de la fonction de Dedekind, $\eta(\tau)=q^{1 / 24} \Pi\left(1-q^{n}\right)$. Plus précisément, on $\mathbf{a}$ :

$$
F(\tau)=\frac{\pi i \tau}{12}-\log \eta(\tau)
$$

On voit en même temps que $F$ est la transformée de Mellin de $\Phi$, c'est-à-dire qu'on a :

$$
\Phi(s)=\int_{0}^{\infty} F(i t) t^{s-1} d t, \quad F(\tau)=\frac{1}{2 \pi i} \int_{\sigma-i \infty}^{\sigma+i \infty} \Phi(s)(\tau / i)^{-s} d s,
$$

la première formule étant valable pour $\operatorname{Re}(s)>1$, et la seconde pour $\sigma>1$, $\operatorname{Im}(\tau)>0$; dans celle-ci, on doit entendre que l'intégrale est prise le long de la droite $\operatorname{Re}(s)=\sigma$. La méthode de Hecke consiste à déplacer cette dernière droite parallèlement à elle-même de manière à l'amener sur la droite $\operatorname{Re}(s)$ $=-\sigma$; en ce faisant, on doit tenir compte des résidus de l'intégrande aux points $s=0, \pm 1$; le comportement de $\Phi$ à l'infini dans la bande $-\sigma \leqq \operatorname{Re}(s) \leqq \sigma$ garantit la légitimité de l'opération, qui donne:

$$
F(\tau)=\frac{\pi i}{12 \tau}-\frac{\pi \tau}{12 i}+\frac{1}{2} \log (\tau / i)+\frac{1}{2 \pi i} \int_{-\sigma-i \infty}^{-\sigma+i \infty} \Phi(s)(\tau / i)^{-s} d s .
$$

En raison de l'équation fonctionnelle obtenue pour $\Phi$, le dernier terme est égal à $F(-1 / \tau)$, d'où en définitive :

$$
\log \eta(-1 / \tau)=\log \eta(\tau)+\frac{1}{2} \log (\tau / i) .
$$

On reconnaît là le résultat classique de Dedekind $([1])$. Bien entendu, on peut eu donner une démonstration plus directe (v. p. ex. [3]). Mais c'est sur le principe appliqué ci-dessus que j'ai surtout voulu attirer l'attention. On aurait $\mathrm{pu}$ traiter exactement de même la fonction $\varphi(s)=\zeta(s) \zeta(s-1)$ (c'est la fonction zêta de l'algèbre simple $M_{2}(\boldsymbol{Q})$ sur le corps $\boldsymbol{Q}$ des rationnels); pour ce choix de $\varphi$, la fonction $\Phi$ définie par la même formule que plus haut satisfait cette fois à l'équation fonctionnelle $\Phi(s)=-\Phi(2-s)$, d'où on déduit le comportement de la transformée de Mellin de $\Phi$, qui est la fonction $F(\tau)=\frac{1}{24}-\frac{1}{2 \pi i} \frac{d}{d \tau} \log \eta(\tau)$. On notera que, dans ces exemples, l'équation fonctionnelle de $\Phi$ ne comporte pas de facteur exponentiel; c'est ce qui permet de les traiter au moyen du 
seul théorème de Hecke. Lorsqu'il en est autrement, il devient nécessaire de mettre en ouvre les moyens supplémentaires que fournit ma note [4].

\section{Bibliographie}

[1] R. Dedekind, Erläuterungen zu den Fragmenten XXVIII, in B. Riemann, Ges. Math. Werke, 2, Aufl., Leipzig 1892, SS. 466-478 (=R. D., Ges. Math. Werke, Bd. I, Vieweg 1930, SS. 159-172).

[2] E. Hecke, Über die Bestimmung Dirichletscher Reihen durch ihre Funktionalgleichung, Math. Ann., 112 (1936), 664-699 (=E.H., Math. Werke, Göttingen 1959, SS. 591-626).

[3] C. L. Siegel, A simple proof of $\eta(-1 / \tau)=\eta(\tau) \sqrt{\tau / i}$, Mathematika, 1 (1954), 4 (=C. L. S., Ges. Abh., Bd. III, Springer 1966, S. 188).

[4] A. Weil, Über die Bestimmung Dirichletscher Reihen durch Funktionalgleichungen, Math. Ann., 168 (1967), 149-156. 